\title{
CONFORMAL DIFFEOMORPHISMS PRESERVING THE RICCI TENSOR
}

\author{
W. KÜHNEL AND H.-B. RADEMACHER
}

(Communicated by Christopher B. Croke)

\begin{abstract}
We characterize semi-Riemannian manifolds admitting a global conformal transformation such that the difference of the two Ricci tensors is a constant multiple of the metric. Unless the conformal transformation is homothetic, the only possibilities are standard Riemannian spaces of constant sectional curvature and a particular warped product with a Ricci flat Riemannian manifold.
\end{abstract}

We consider semi-Riemannian manifolds $(M, g)$ and conformal diffeomorphisms $f:(M, g) \rightarrow(\bar{M}, \bar{g})$ between them meaning that $f^{*} \bar{g}$ is pointwise a positive scalar multiple of $g$. If this factor is constant $f$ is called a homothety. A conformal structure on $M$ is a class of conformally equivalent metrics. In this paper we study the behavior of the Ricci tensor $\mathrm{Ric}_{g}$ within one conformal class A classical theorem of Liouville determines all possible conformal diffeomorphisms between euclidean metrics. As a generalization we call a conformal transformation $g \rightarrow \bar{g}$ a Liouville transformation if $\mathrm{Ric}_{\bar{g}}-\mathrm{Ric}_{g}=0$. In Theorems 1 and 2 we classify complete semi-Riemannian manifolds $(M, g)$ admitting a non-homothetic conformal transformation $\bar{g}=\varphi^{-2} g$ such that the difference $\mathrm{Ric}_{\bar{g}}-\mathrm{Ric}_{g}$ of the Ricci tensors is a constant multiple of the metric $g$ or $\bar{g}$. We show that $M$ is Riemannian and that $M$ is either a standard space of constant sectional curvature or is a warped product $\mathbb{R} \times_{\exp } M_{*}$ of the real line and a Ricci flat manifold $M_{*}$. As a special case we obtain in Corollary 1 that a globally defined Liouville transformation is a homothety. This result is due to Liouville [Liv] in the case of $E^{3}$, generalized by Lie [Lie] to the case of $E^{n}$ (see also [S, p. 173]) and by Haantjes [H] to the case of pseudo-euclidean space $E_{k}^{n}$. For Riemannian manifolds Corollary 1 has been obtained by Ferrand [Fe], where a Liouville transformation is called a quasisimilarity. For the compact Riemannian case, compare the recent paper [X] but be careful with the signs in formula (2) there. In Theorem 3 we show that for a complete semi-Riemannian manifold admitting a global non-homothetic concircular transformation between two metrics of constant scalar curvature the same conclusions as in Theorem 1 hold.

Received by the editors August 25, 1993 and, in revised form, February 3, 1994.

1991 Mathematics Subject Classification. Primary 53C20, 53C50; Secondary 53A30, 58 G30.

Key words and phrases. Semi-Riemannian manifolds, Ricci tensor, conformal mapping, Hessian. 
General assumption. $\left(M^{n}, g\right)$ is a semi-Riemannian manifold of dimension $n \geq 3$ and of class $C^{3}$ such that the number of negative eigenvalues of $g$ is not greater than $\frac{n}{2}$.

Notation. $\operatorname{grad} \varphi$ denotes the gradient of $\varphi, \nabla^{2} \varphi=\nabla \operatorname{grad} \varphi$ is the Hessian of $\varphi$, and $\Delta \varphi=$ trace $\nabla^{2} \varphi$ is the Laplacian. Let us introduce the notation $[h]$ for the class of all tensors which are pointwise scalar multiples of a given $(0,2)$-tensor $h$. This includes the zero tensor-as well as negative multipleswhich is not in the conformal structure itself. $(M, g)$ is called an Einstein space if $\left[\mathrm{Ric}_{g}\right]=[g]$. Two metrics $g, \bar{g}$ are concircular to one another if $[g]=[\bar{g}]=\left[\mathrm{Ric}_{\bar{g}}-\mathrm{Ric}_{g}\right]$.

Lemma 1. Two conformally equivalent metrics $g$ and $\bar{g}=\frac{1}{\varphi^{2}} g$ satisfy the relation

$$
\left[\mathrm{Ric}_{\bar{g}}-\mathrm{Ric}_{g}\right]=[g]=[\bar{g}]
$$

if and only if the function $\varphi$ satisfies the equation

$$
\nabla^{2} \varphi=\frac{\Delta \varphi}{n} \cdot g
$$

Proof. This follows from the well-known formula [S, p. 168], [Be, Sect. 1J], [Kü, Sect. A]

$$
\operatorname{Ric}_{\bar{g}}-\operatorname{Ric}_{g}=\frac{1}{\varphi^{2}}\left[(n-2) \varphi \nabla^{2} \varphi+(\varphi \Delta \varphi-(n-1) g(\operatorname{grad} \varphi, \operatorname{grad} \varphi)) \cdot g\right] .
$$

Lemma 1 holds only under the assumption $n \geq 3$.

Remark. A transformation $g \rightarrow \bar{g}$ as in Lemma 1 is called concircular because it preserves the curves of constant geodesic curvature and vanishing geodesic torsion (so-called geodesic circles); see [Y]. In this case the conformal geodesics are geodesic circles; see [Fi, p. 454]. Any conformal transformation between two Einstein spaces is automatically concircular. A concircular transformation $g \rightarrow \bar{g}$ satisfies $\operatorname{Ric}_{\bar{g}}-\operatorname{Ric}_{g}=\frac{1}{n}\left(\frac{\bar{S}}{\varphi^{2}}-S\right) \cdot g$ where $S, \bar{S}$ denote the scalar curvatures of $g, \bar{g}$.

Lemma 2. A function $\varphi: M \rightarrow \mathbb{R}$ satisfies $\nabla^{2} \varphi=\lambda \cdot g$ for some $\lambda: M \rightarrow \mathbb{R}$ in a neighborhood of a point with $g(\operatorname{grad} \varphi, \operatorname{grad} \varphi) \neq 0$ if and only if $g$ is locally a warped product metric $d s^{2}=\eta d t^{2}+\varphi^{2}(t) d s_{*}^{2}$, where $\eta \in\{+1,-1\}$ denotes the sign of $g(\operatorname{grad} \varphi, \operatorname{grad} \varphi), \varphi, \lambda$ are functions depending only on $t$ satisfying $\varphi^{\prime \prime}=\eta \cdot \lambda$, and $d s_{*}^{2}$ is independent of $t$.

This lemma can be found in [Fi, Sect. 12] and also in [T] for the Riemannian case. For the special case of Einstein metrics it is due to Brinkmann [Br].

Particular consequences of Lemma 2 are the following:

1. If $\nabla^{2} \varphi=\lambda g$ and if $\operatorname{grad} \varphi$ is a non-null vector, then the trajectories of $\operatorname{grad} \varphi$ are geodesics (up to parametrization).

2. If $\nabla^{2} \varphi=\lambda g$, then along every such non-null geodesic $\gamma(t)$ in direction $\operatorname{grad} \varphi$ with $g(\dot{\gamma}, \dot{\gamma})=\eta$ the function $\varphi(t):=\varphi(\gamma(t))$ satisfies $\varphi^{\prime \prime}=\eta \cdot \lambda$. Along a null geodesic this function $\varphi(t)$ satisfies $\varphi^{\prime \prime}=0$ according to the proof of Lemma 3 below. 
Definition. A semi-Riemannian manifold $(M, g)$ is called (geodesically) complete if every geodesic can be defined over $\mathbb{R}[\mathrm{O}, \mathrm{p}$. 68]. It is called null complete if this holds for every null geodesic.

Theorem 1. Let $(M, g)$ be complete and admitting a global conformal transformation $\bar{g}=\frac{1}{\varphi^{2}} \cdot g$ satisfying

$$
\operatorname{Ric}_{\bar{g}}-\operatorname{Ric}_{g}=c \cdot(n-1) \cdot g
$$

for some constant $c$. Then one of the following three cases occurs:

1. $\varphi$ is constant.

2. $(M, g)$ and $(M, \bar{g})$ are simply connected Riemannian spaces of constant sectional curvature.

3. $(M, g)$ is a warped product $\mathbb{R} \times_{e^{t}} M_{*}$ where $\varphi(t)=e^{t}$ and $\left(M_{*}, g_{*}\right)$ is a complete Ricci-flat $(n-1)$-dimensional Riemannian manifold.

Remark. In Theorem 1 case 1 corresponds to $c=0$, and cases 2 and 3 correspond to $c>0$. In case $2 \bar{g}$ must be flat and $g$ must be hyperbolic, and in case $3 \operatorname{Ric}_{\bar{g}}=0$. A non-constant $\varphi$ occurs only for Einstein space. Therefore we compare this to another theorem.

Theorem $1^{*}$. Let $(M, g)$ be complete and assume that both $g$ and $\bar{g}=\frac{1}{\varphi^{2}} \cdot g$ are Einstein metrics. Then the same conclusion as in Theorem 1 holds, i.e. one of the cases 1, 2, 3 occurs.

Remark. In Theorem $1^{*}$ case 2 occurs for various combinations of the signs of the constant curvatures of $g$ and $\bar{g}$.

Theorem 2. Let $(M, g)$ be complete and admitting a global conformal transformation $\bar{g}=\frac{1}{\varphi^{2}} \cdot g$ satisfying

$$
\operatorname{Ric}_{\bar{g}}-\operatorname{Ric}_{g}=c \cdot(n-1) \cdot \bar{g}=\frac{c \cdot(n-1)}{\varphi^{2}} \cdot g
$$

for a constant $c$. Then either $\varphi$ is constant or $(M, g)$ is isometric with the euclidean space.

Remark. To pass from Theorem 1 to Theorem 2 one just has to interchange the roles of $g$ and $\bar{g}$. However, this is not quite symmetric because at most one of them can be complete (unless $\varphi$ is constant). In Theorem 2 a constant $\varphi$ corresponds to $c=0$; the other case corresponds to $c>0$. In particular, $c<0$ is impossible in Theorem 1 and in Theorem 2 .

Corollary 1. A globally defined Liouville transformation of a complete semiRiemannian manifold is a homothety.

This result is just the case $c=0$ in Theorems 1 and 2 .

Corollary 2. Assume that two semi-Riemannian metrics in the same conformal class have pointwise the same Ricci tensor. If one of them is complete, then they are homothetic to one another.

This is a uniqueness result for the problem of prescribing a Ricci tensor in a conformal class. 
Lemma 3. Let $g$ be a null complete indefinite metric admitting a globally defined nonconstant solution $\varphi$ of $\nabla^{2} \varphi=\frac{\Delta \varphi}{n} \cdot g$. Then $\varphi$ has a zero.

Proof. Along any null geodesic $\gamma(s)$ one calculates

$$
\begin{aligned}
\frac{d^{2}}{d s^{2}}(\varphi(\gamma(s))) & =\frac{d}{d s} g(\operatorname{grad} \varphi, \dot{\gamma}) \\
& =g\left(\nabla_{\dot{\gamma}} \operatorname{grad} \varphi, \dot{\gamma}\right)+g\left(\operatorname{grad} \varphi, \nabla_{\dot{\gamma}} \dot{\gamma}\right) \\
& =g\left(\frac{\Delta \varphi}{n} \cdot \dot{\gamma}, \dot{\gamma}\right) \\
& =0 .
\end{aligned}
$$

Therefore $\varphi(\gamma(s))$ is linear in $s$ with $\frac{d}{d s} \varphi(\gamma(s))=g(\operatorname{gradf} \varphi, \dot{\gamma})$. If we choose $\gamma$ such that $g(\operatorname{grad} \varphi, \dot{\gamma}) \neq 0$ at a point $p$, then it follows that $\varphi$ has a zero along $\gamma$.

Corollary 3. The only globally defined concircular transformations of a null complete indefinite metric are the homotheties.

Theorem 3. Let $(M, g)$ be complete and admitting a globally defined concircular transformation $\bar{g}=\frac{1}{\varphi^{2}} \cdot g$. Assume that $S, \bar{S}$ are constant. Then one of the three cases 1, 2, 3 as in Theorem 1 occurs.

Proof of Theorem 1. By assumption $\varphi: M \rightarrow \mathbb{R}$ is a function which is positive everywhere. By Lemma 3 we may assume that $\varphi$ is a non-constant function on a manifold with positive definite metric $g$. Let $p \in M$ be a point with $\operatorname{grad} \varphi(p) \neq 0$. By Lemma 1 the equation

$$
\begin{aligned}
c(n-1) \cdot g & =\operatorname{Ric}_{\bar{g}}-\operatorname{Ric}_{g} \\
& =\frac{1}{\varphi^{2}}\left[(n-2) \varphi \cdot \nabla^{2} \varphi+\left(\varphi \Delta \varphi-(n-1)\|\operatorname{grad} \varphi\|^{2}\right) \cdot g\right]
\end{aligned}
$$

implies

$$
\frac{2(n-1)}{n} \varphi \Delta \varphi-(n-1)\|\operatorname{grad} \varphi\|^{2}-c(n-1) \varphi^{2}=0 .
$$

By Lemma 2 along the unit speed geodesic $\gamma(t)$ in the direction of $\operatorname{grad} \varphi=$ $\varphi^{\prime} \cdot \frac{\partial}{\partial t}$ we have

$$
2 \varphi \varphi^{\prime \prime}-\varphi^{\prime 2}-c \varphi^{2}=0
$$

Differentiating once more we get

$$
2 \varphi \varphi^{\prime \prime \prime}-2 c \varphi \varphi^{\prime}=0
$$

or, since $\varphi \neq 0$,

$$
\varphi^{\prime \prime \prime}=c \cdot \varphi^{\prime} .
$$

Therefore there is a constant $a$ satisfying

$$
\varphi^{\prime \prime}=c \cdot \varphi+a .
$$

$\varphi^{\prime}(p) \neq 0$ implies equivalently

$$
\left(\varphi^{\prime 2}\right)^{\prime}=c \cdot\left(\varphi^{2}\right)^{\prime}+2 a \varphi^{\prime} .
$$


This means that there is a constant $b$ satisfying

$$
\varphi^{\prime 2}=c \cdot \varphi^{2}+2 a \varphi+b .
$$

Now (5), (7), and (2) together imply $b=0$; thus

$$
\varphi^{\prime 2}=\varphi(c \cdot \varphi+2 a) .
$$

Case I: $c=0$. By (5) $\varphi$ is a polynomial of degree at most 2. Then (8) reduces to $\varphi^{\prime 2}=2 a \varphi$, which implies that $\varphi$ is quadratic and that $\varphi$ and $\varphi^{\prime}$ have a common zero along $\gamma$. By the completeness of $g$ the metric $\bar{g}=\frac{1}{\varphi^{2}} \cdot g$ has a singularity there, a contradiction. Alternatively, if $\varphi(t)=A t^{2}+B t+C$, then (8) implies that the discriminant $4 A C-B^{2}$ is zero. Therefore $\varphi(t)$ is the square of a linear function.

Case II: $c<0$. In this case every solution $\varphi$ of $(5)$ is periodic and therefore attains its minimum and maximum. At each of these points the equation $0=$ $\varphi(c \varphi+2 a)$ is satisfied by (8). Hence $\varphi=0$ and $c \varphi+2 a=0$ must be satisfied at the minimum and maximum, respectively. This leads to a contradiction as in Case I.

Alternatively, the general solution of (5)

$$
\varphi(t)=\alpha \cos (\sqrt{-c} t)+\beta \sin (\sqrt{-c} t)-\frac{a}{c}
$$

satisfies

$$
\alpha^{2}+\beta^{2}=\frac{a^{2}}{c^{2}}
$$

by (8). A typical solution looks like $\varphi(t)=\cos t+1$.

Case III: $c>0$. In this case the general solution of (5) is

$$
\varphi(t)=\alpha \cosh (\sqrt{c} t)+\beta \sinh (\sqrt{c} t)-\frac{a}{c} .
$$

Then (8) implies

$$
\alpha^{2}-\beta^{2}=\frac{a^{2}}{c^{2}}
$$

in particular $\alpha^{2} \geq \beta^{2}$.

If $\alpha^{2}>\beta^{2}$, then $\varphi$ has a critical point along $\gamma$. This implies that $\gamma$ has a point $q$ with $\operatorname{grad} \varphi(q)=0$ (note that $\operatorname{grad} \varphi=\varphi^{\prime} \cdot \frac{\partial}{\partial t}$ ). Furthermore $\varphi$ satisfies globally $\nabla^{2} \varphi=(c \varphi+a) \cdot g$ with $c>0$. A result of Tashiro [T] implies that $(M, g)$ is isometric with the hyperbolic space of constant sectional curvature $-c$. Roughly the argument is the following: In geodesic polar coordinates around $q$ the metric $g$ looks like $d s^{2}=d t^{2}+\sinh ^{2}(\sqrt{c} t) \cdot d s_{1}^{2}$ where $d s_{1}^{2}$ is the metric of a round sphere of appropriate radius; compare [Kü, Lemma 18].

If $\alpha^{2}=\beta^{2}$, then $a=0$ and $\varphi(t)=\alpha \cdot e^{\mp \sqrt{c} \cdot t}$ is a solution without a critical point along $\gamma$. This implies that

$$
d s^{2}=d t^{2}+e^{2 \sqrt{c} \cdot t} d s_{*}^{2}
$$

is a complete metric on $M=\mathbb{R} \times M_{*}$. It follows that $\bar{g}=e^{-2 \sqrt{c t} g}$ is the product metric $d t^{2}+d s_{*}^{2}$ on $(0, \infty) \times M_{*}$. For an arbitrary tangent vector 
$X$ in the direction of $M_{*}$ the standard formulas for the curvature of warped products imply

$$
\begin{aligned}
c(n-1) g(X, X) & =\left(\operatorname{Ric}_{\bar{g}}-\operatorname{Ric}_{g}\right)(X, X) \\
& =\left(1-c \cdot e^{2 \sqrt{c} t}\right) \operatorname{Ric}_{g_{*}}(X, X)+c(n-1) g(X, X),
\end{aligned}
$$

which is impossible unless $\operatorname{Ric}_{g_{*}}=0$. This completes the proof of Theorem 1 .

Note that in the case of an indefinite metric (9) does not define a complete warped product metric; compare $[\mathrm{O}$, p. 209]. Compare also $[\mathrm{Kb}]$ for global solutions of $\nabla^{2} \varphi=c \cdot \varphi \cdot g, c>0$, in the indefinite case if $\varphi$ has at least one critical point.

Proof of Theorem $1^{*}$. In the case of indefinite metrics $\varphi$ is constant by Lemma 3 , using the same argument as in Theorem 1. The Riemannian case has been treated in [Kü, Theorem 27]. The local considerations in this case are due to Brinkmann [Br]. Compare also [Be, 9.110].

Remark. Geodesic mappings of the same kind as in Theorem 1 have been studied in [V]. For the case of conformal vector fields on Einstein spaces compare [YN] and [Kan] in the Riemannian case and [Ke1], [Ke2] in the nonRiemannian case. Brinkmann describes in $[\mathrm{Br}, \S 4]$ indefinite Einstein metrics $(M, g)$ carrying a non-constant positive function $\varphi$ such that the conformally equivalent metric $\bar{g}:=\varphi^{-2} g$ is also Einstein and the gradient $\operatorname{grad} \varphi$ is everywhere null. Then it follows that $\operatorname{Ric}_{\bar{g}}=\operatorname{Ric}_{g}=0$ and $\nabla^{2} \varphi=0$, i.e. $\operatorname{grad} \varphi$ is parallel. By Lemma $3(M, g)$ cannot be null complete.

In general relativity these metrics were studied in several papers. They are called pp-waves or gravitational plane waves; see e.g. [Hf].

Locally all the considerations in the proofs of Theorems 1-3 remain valid also in the case of an indefinite metric. This includes a local classification and the existence of various examples which, however, cannot be null complete.

Proof of Theorem 2. This follows the pattern of the proof of Theorem 1. In particular $g$ must be positive definite if $\varphi$ is not constant. We start with the equation

$\frac{c \cdot(n-1)}{\varphi^{2}} \cdot g=\operatorname{Ric}_{\bar{g}}-\operatorname{Ric}_{g}=\frac{1}{\varphi^{2}}\left[(n-2) \varphi \cdot \nabla^{2} \varphi+\left(\varphi \Delta \varphi-(n-1)\|\operatorname{grad} \varphi\|^{2}\right) \cdot g\right]$

which implies

$$
\frac{2(n-1)}{n} \varphi \Delta \varphi-(n-1)\|\operatorname{grad} \varphi\|^{2}-c(n-1)=0 .
$$

If $\operatorname{grad} \varphi \neq 0$ at $p$, then along the geodesic $\gamma$ in direction $\operatorname{grad} \varphi$ we have

$$
2 \varphi \varphi^{\prime \prime}-\varphi^{\prime 2}-c=0,
$$

which implies

$$
2 \varphi \varphi^{\prime \prime \prime}=0
$$

or

$$
\varphi(t)=A t^{2}+B t+C .
$$

If we put this into (11) we get

$$
4 A C-B^{2}=c .
$$


The case $c=0$ leads to a zero of $\varphi$ as in the proof of Theorem 1; the case $c<0$ leads to two zeros of $\varphi$, a contradiction. If $c>0$, then $\varphi$ has no zero but it has a critical point along $\gamma$. This is a critical point for $\varphi$ on $M . \varphi$ satifies the equation $\nabla^{2} \varphi=2 A \cdot g$. By a theorem of Tashiro [T] this implies that $(M, g)$ is isometric with the euclidean space. Around the critical point the geodesic polar coordinates coincide with the euclidean polar coordinates.

In particular, if $\varphi$ is non-constant, then $c$ must be positive and $\bar{g}$ is a space of constant sectional curvature $c$.

Proof of Theorem 3. The case of an indefinite metric can be ruled out by Lemma

3. In the Riemannian case the equation

$$
\operatorname{Ric}_{\bar{g}}-\operatorname{Ric}_{g}=\frac{1}{n}\left(\frac{\bar{S}}{\varphi^{2}}-S\right) \cdot g
$$

implies

$$
2 \varphi \varphi^{\prime \prime}-\varphi^{2}+\frac{S}{n(n-1)} \cdot \varphi^{2}-\frac{\bar{S}}{n(n-1)}=0
$$

along a unit speed geodesic in direction $\operatorname{grad} \varphi$. Differentiating once more leads to

$$
2 \varphi \varphi^{\prime \prime \prime}+\frac{2 S}{n(n-1)} \varphi \varphi^{\prime}=0
$$

or

$$
\varphi^{\prime \prime \prime}+\rho \cdot \varphi^{\prime}=0
$$

where $\rho:=\frac{S}{n(n-1)}$ denotes the normalized scalar curvature.

As in the proof of Theorem 1 we conclude

$$
\varphi^{\prime 2}=-\rho \varphi^{2}+2 a \cdot \varphi-\bar{\rho}
$$

for a certain constant $a$.

In any case the solution $\varphi$ of (17) and (18) either has a zero (which is impossible because $\varphi$ is a conformal factor) or a critical point, except for solutions of the type

$$
\varphi^{\prime}(t)=\alpha \cdot e^{\sqrt{-\rho} t}
$$

leading to the same warped product metric as in (9). If there is a critical point, then the levels around it are round spheres and thus $(M, g)$ is a standard space of constant sectional curvature [T], [Kü, Lemmas 13 and 18]. This completes the proof of Theorem 3 .

The local part of this calculation is due to Tachibana [Tb, Theorem 8.1]. In the compact case the following holds: a compact Riemannian manifold with constant scalar curvature admitting a non-constant solution of

$$
\nabla^{2} \varphi=\frac{\Delta \varphi}{n} \cdot g
$$

is isometric with a round sphere [Kü, Theorem 24]. 


\section{REFERENCES}

[Be] A. L. Besse, Einstein manifolds, Ergebnisse Math. Grenzgeb., 3. Folge, Band 10, Springer, Berlin, Heidelberg, and New York, 1987.

[Br] H. W. Brinkmann, Einstein spaces which are mapped conformally on each other, Math. Ann. 94 (1925), 119-145.

[Fe] J. Ferrand, Sur une classe de morphismes conformes, C. R. Acad. Sci. Paris 296 (1983), 681-684.

[Fi] A. Failkow, Conformal geodesics, Trans. Amer. Math. Soc. 45 (1939), 443-473.

[H] J. Haantjes, Conformal representations of an n-dimensional euclidean space with a nondimensional euclidean space with a non-definite fundamental form on itself, Proc. Kon. Nederl. Akad. Amsterdam 40 (1937), 700-705.

[Hf] W. D. Halford, Brinkmann's theorem in general relativity, Gen. Relativity Gravitation 14 (1982), 1193-1195.

[Kan] M. Kanai, On a differential equation characterizing a Riemannian structure of a manifold, Tokyo J. Math. 6 (1983), 143-151.

[Kb] Y. Kerbrat, Transformations conformes des variétés pseudo-Riemanniennes, J. Differential Geom. 11 (1976), 547-571.

[Ke1] M. G. Kerckhove, Conformal transformations of pseudo-Riemannian Einstein manifolds, thesis, Brown Univ., 1988.

[Ke2] _ The structure of Einstein spaces admitting conformal motions, Classical Quantum Gravity 8 (1991), 819-825.

[Kü] W. Kühnel, Conformal transformations between Einstein spaces, Conformal Geometry ( $\mathbf{R}$. S. Kulkarni and U. Pinkall, eds.). Aspects of Math., vol. E12, Braunschweig, 105-146, Vieweg-Verlag, 1988, pp. 105-146.

[Lie] S. Lie, Komplexe, insbesondere Linien und Kugelkomplexe mit Anwendung auf die Theorie partieller Differentialgleichungen, Math. Ann. 5 (1872), 145-246.

[Liv] J. Liouville, Extension au cas des trois dimensions de la question du tracé géographique, Note VI, Applications de l'Analyse à la Géométrie (G. Monge, ed.), Paris, 1850, pp. 609-617.

[O] B. O'Neill, Semi-Riemannian geometry with applications to relativity, Academic Press, New York, 1983.

[S] J. A. Schouten, Der Ricci-Kalkül, Springer-Verlag, Berlin, 1924.

[Tb] S. Tachibana, On concircular geometry and Riemann spaces with constant scalar curvatures, Tôhoku Math. J. 3 (1951), 149-158.

[T] Y. Tashiro, Complete Riemannian manifolds and some vector fields, Trans. Amer. Math. Soc. 117 (1965), 251-275.

[V] P. Venzi, Klassifikation der geodätischen Abbildungen mit $\overline{\mathrm{Ric}}-\mathrm{Ric}=\Delta \cdot g$, Tensor (N.S.) 37 (1982), 137-147.

[X] X. Xu, Prescribing a Ricci tensor in a conformal class of Riemannian metrics, Proc. Amer. Math. Soc. 115 (1992), 455-459; corrigenda, ibid. 118 (1993), 333.

[Y] K. Yano, Concircular geometry I-V, Proc. Imperial Acad. Japan 16 (1940), 195-200, 354360, 442-448, 505-511; ibid. 18 (1942), 446-451.

[YN] K. Yano and T. Nagano, Einstein spaces admitting a one-parameter group of conformal transformations, Ann. of Math. (2) 69 (1959), 451-460.

Fachbereich Mathematik, Universität Duisburg, 47048-Duisburg, Germany

Current address: Mathematisches Institut B, Universität Stuttgart, 70550 Stuttgart, Germany

E-mail address: kuehnel@morse.physik.uni-stuttgart.de

Institut für Mathematik, Universität Augsburg, 86135-Augsburg, Germany Current address: Mathematisches Institut, Universität Leipzig, 04009 Leipzig, Germany

E-mail address: rademacher@uni-augsburg.de 